\title{
Robust phase unwrapping based on non-coprime fringe pattern periods for deflectometry measurements
}

Allgeier, Stephan, Gengenbach, Ulrich, Köhler, Bernd, Reichert, Klaus-Martin, Hagenmeyer, Veit

Stephan Allgeier, Ulrich Gengenbach, Bernd Köhler, Klaus-Martin Reichert, Veit Hagenmeyer, "Robust phase unwrapping based on non-coprime fringe pattern periods for deflectometry measurements," Proc. SPIE 11061, Automated Visual Inspection and Machine Vision III, 1106103 (21 June 2019); doi: $10.1117 / 12.2523564$

SPIE. Event: SPIE Optical Metrology, 2019, Munich, Germany 


\title{
Robust phase unwrapping based on non-coprime fringe pattern periods for deflectometry measurements
}

\author{
Stephan Allgeier, Ulrich Gengenbach, Bernd Köhler, Klaus-Martin Reichert, and Veit \\ Hagenmeyer \\ Institute for Automation and Applied Informatics, Karlsruhe Institute of Technology (KIT), \\ Hermann-von-Helmholtz-Platz 1, 76344 Eggenstein-Leopoldshafen, Germany
}

\begin{abstract}
Phase-measuring deflectometry is a technique for non-contact inspection of reflective surfaces. A camera setup captures the reflection of a sine-modulated fringe pattern shifted across a screen; the location-dependent measured phase effectively encodes the screen coordinates. As the used fringe patterns are much narrower than the screen dimension, the resulting phase maps are wrapped. The number-theoretical solution uses the Chinese remainder theorem to calculate an unwrapped phase map from repeated measurements with coprime fringe widths. The technique is highly susceptible to phase noise, i.e. small deviations of the measured phase values generally lead to unwrapped phase values with large errors. We propose a modification and show how non-coprime period widths make phase unwrapping robust against phase noise. Measurements with two non-coprime fringe period widths introduce the opportunity to discriminate between "legal" measured phase value pairs, that potentially originate from noise-free measurements, and "illegal" phase value pairs, that necessarily result from noise-affected measurements. Arranged as a matrix, the legal measurements lie on distinct diagonals. This insight not only allows to determine the legality of a measurement, but also to provide a correction by looking for the closest legal matrix entry. We present an experimental comparison of the resulting phase maps with reference phase maps. The presented results include descriptive statistics on the average rate of illegal phase measurements as well as on the deviation from the reference. The measured mean absolute deviation decreases from 1.99 pixels before correction to 0.21 pixels after correction, with a remaining maximum absolute deviation of 0.91 pixels.
\end{abstract}

Keywords: printed electronics, surface inspection, deflectometry, phase unwrapping, phase noise correction

\section{INTRODUCTION}

Functional printing is a promising technology to fabricate electronic structures on flexible, conformable or even stretchable substrates. Due to its additive processes it offers the potential to realize resource efficient and environmentally friendly fabrication. The multitude of available printing processes allows to span a large range of production volumes. Small volumes of e.g. laterally structured devices like wearables, customized to the individual, can be fabricated by ink-jet printing techniques, while large area structures such as solar cells can be produced by roll-to-roll manufacturing in large volume. An additional benefit of functional printing is the continuously growing range of inks based on novel nanomaterials that allow to print conductors, passive electronic components (e.g. resistors, capacitors) and active electronic components (e.g. diodes, transistors, photodiodes, sensors). ${ }^{1,2}$ Quality and yield of printed electronic devices depend, among other factors, on the interaction between printing system, ink and substrate. A constant distance $(0.5 \mathrm{~mm}-1.0 \mathrm{~mm})$ between substrate and print head is paramount for reproducible ink-jet printing results. Hence, planarity of the substrate becomes a significant factor. Established substrate materials in functional printing are polymer foils such as polyethylene terephthalate (PET) for low temperatures and low-cost applications or polyimide (PI) for higher temperature process and application ranges. The substrates usually have good surface quality but are supplied with embedded stresses from the fabrication process that may lead to substrate warpage. In multistage printing processes with multiple thermal post-processing steps (e.g. drying, sintering), additional stresses can be embedded in the substrate that may lead to additional local substrate warpage. These substrate deformations have to be detected in order to

Corresponding author: Stephan Allgeier, stephan.allgeier@kit.edu 


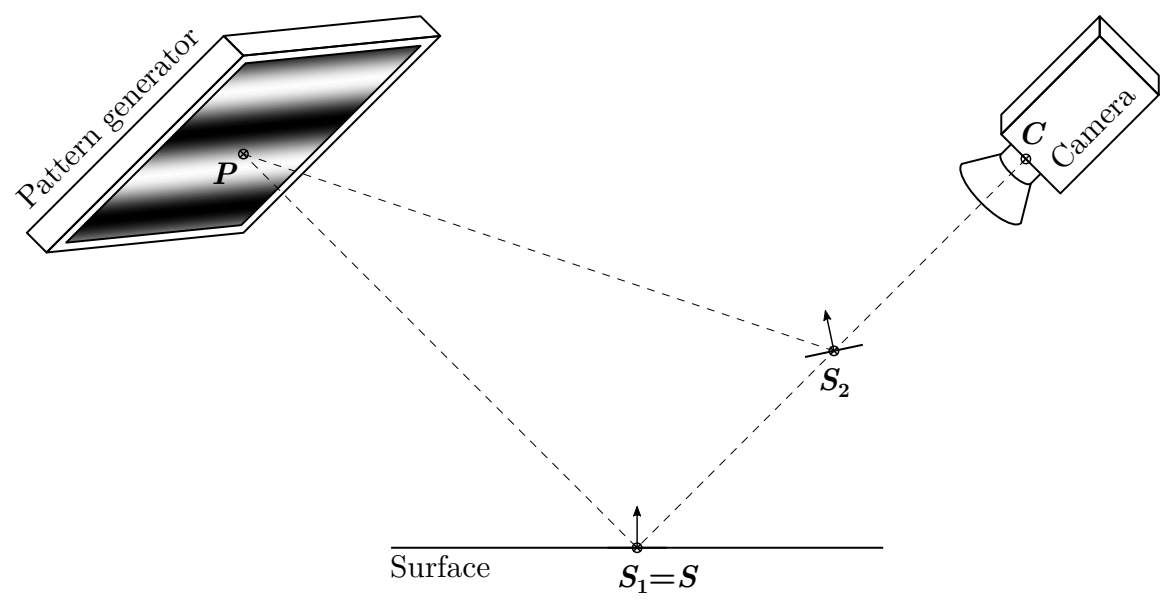

Figure 1. Measurement principle of phase-measuring deflectometry: 1. The camera captures the presented patterns, reflected by the surface under test. 2. Correspondences $(C, P)$ are established by using position-encoding patterns. 3. Each potential surface point (e.g. $S_{1}$ or $S_{2}$ ) on the the view ray of camera pixel $C$ possesses a distinct normal vector, which is the vector bisecting the angle between $\overrightarrow{S_{i} C}$ and $\overrightarrow{S_{i} P}$; additional information is required to discriminate the real surface point $S$ from among the $S_{i}$ (see text).

optimize substrate clamping or active adjustment of print head distance. As the substrates are reflective and frequently transparent, phase-measuring deflectometry ${ }^{3,4}$ (PMD) can be used for non-contact inspection of their surface topography.

The operating principle of PMD can be summarized as follows. A camera setup is directed at the reflective surface to be inspected (cf. Fig. 1). Due to its reflective and transparent nature, the surface itself is basically invisible to the camera. Instead, the camera captures the reflection of sine-modulated fringe patterns that move across a screen (or more generally a pattern generator) along specific directions. The phase of the locationdependent intensity function effectively encodes the screen coordinates. Two-dimensional patterns that encode both screen dimensions at the same time have been proposed,${ }^{5-7}$ but currently the more commonly implemented approach is to use separate fringe patterns to encode the horizontal and the vertical screen coordinates, respectively. ${ }^{3}$ Measuring the pattern phases and decoding the corresponding screen coordinates from an image sequence of the reflected moving patterns establishes a correspondence between each camera pixels and the screen coordinates it captures. Finally, by tracing the view ray from a camera pixel onto the reflective surface and from there to the corresponding screen location, topographical information on the measured surface (primarily on its normal vectors) can be inferred. In this last step, the location of the surface point on a view ray can not be derived from a single pixel-to-screen-correspondence alone, but requires additional information; this is sometimes called the height problem ${ }^{8}$ or the height-slope ambiguity ${ }^{4}$ in PMD. A variety of solutions has been proposed, ${ }^{9,10}$ ranging from repeated measurements with defined movement of the pattern generator ${ }^{11}$ or camera, simultaneous additional measurements using stereo or multi-camera setups, ${ }^{3}$ introduction of known surface points from additional sensor information, to specifying mathematical constraints on the surface topography. ${ }^{9}$

The fringe width of the fringe pattern is the main influencing factor on the effective local resolution of the measurements, therefore fringes as narrow as practically possible are favorable. ${ }^{12}$ The major constraint for that practical limit is that the fringes have to be imaged with good contrast by the camera setup. As the fringe patterns used in practice are therefore much narrower than the screen dimension, the resulting phase maps are commonly wrapped. Different algorithmic techniques to derive the unambiguous unwrapped phase maps have been published, both local ones that unwrap a single phase map, ${ }^{13}$ and others that process two or more phase maps, acquired with different fringe pattern periods. ${ }^{14}$ The latter are called temporal methods because they require multiple measurements of the same scene. One solution, the number-theoretical approach, ${ }^{14}$ is to use two coprime fringe period widths and calculate an unwrapped phase map using the Chinese remainder theorem. The technique is highly susceptible to phase noise, i.e. small deviations of the measured phase values generally lead to unwrapped phase values with large errors. ${ }^{14}$ As the unwrapped phase values are essentially the screen 

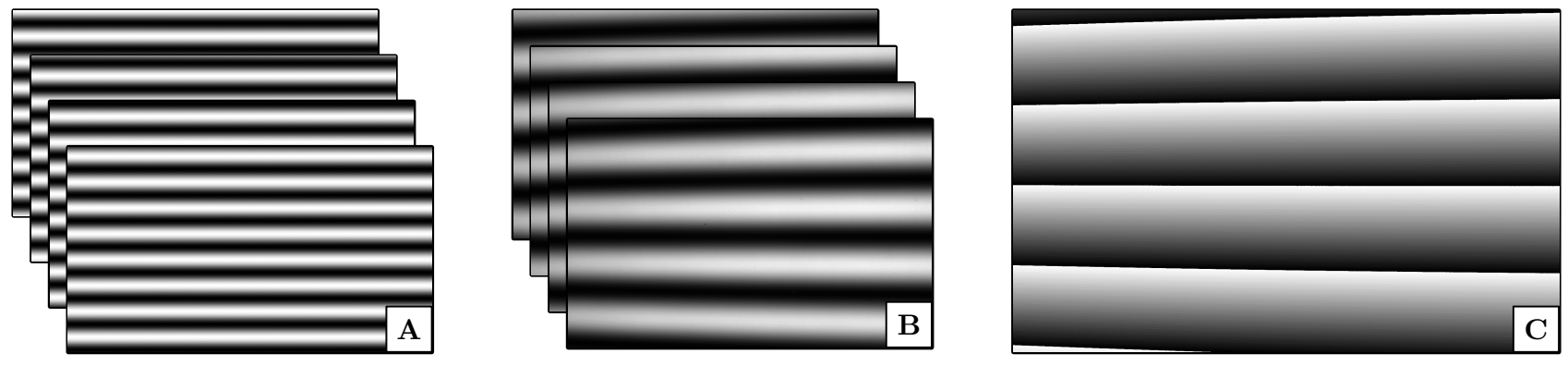

Figure 2. (A) Sine-modulated fringe patterns encode the screen coordinates; the shown pattern with a fringe width of $\omega=272$ pixels encodes the vertical screen dimension (the screen size is $3840 \times 2160$ pixels). (B) The reflected pattern captured by the camera may be distorted due to perspective and the curvature of the measured surface. (C) The phase map computed from the captured images is wrapped, because the used fringe width is much smaller then the screen dimensions.

coordinates used for the camera-to-screen-correspondences and the calculation of the surface normals, these errors have large detrimental effects on the reconstructed surface topographies. In the present contribution, we propose a modification to the number-theoretical approach using non-coprime period widths.

In Sec. 2.1 we briefly describe the phase retrieval process in PMD. Section 2.2 begins with a description the number-theoretical approach to phase unwrapping with coprime fringe pattern periods and explains the method's high susceptibility to phase noise. We then introduce the effects of using fringe patterns with non-coprime periods and how they can be exploited to detect and correct phase noise errors. Section 3 describes the setup and results of an experimental comparison of the suggested method with a reference method. In Sec. 4 we conclude with a brief discussion of the results.

\section{METHODS}

Before detailing the proposed phase unwrapping algorithm, we provide a short explanation of the phase retrieval process in PMD, i.e. the calculation of the (wrapped) phase maps from the acquired image data. The following descriptions will only consider fringe patterns - and resulting phase maps - that encode a single dimension of the screen. The process is usually repeated with perpendicular fringe patterns to encode the other screen dimension, all subsequent calculations are identical.

\subsection{Phase Retrieval}

Consider a fringe pattern encoding the vertical screen dimension, as seen in Fig. 2A. The fringe pattern can be described as

$$
I_{s}(u, v, t)=a_{s}+b_{s} \cos \left(2 \pi \frac{v}{\omega}-2 \pi \frac{t}{n}\right), \text { with } t=0, \ldots, n-1,
$$

where $a_{s}$ is the mean intensity value, $b_{s}$ is the intensity amplitude (measured from mean to peak value), and $\omega$ is the fringe pattern period width, given in the same unit as the pattern location variables $u$ and $v$. We will assume this pattern unit to be screen pixels in the following description, but arbitrary other units are perfectly valid as long as they allow the integer arithmetic of the number-theoretical phase unwrapping to be carried out. The integer $t$ describes the vertical shift of the pattern in $n$ discrete time steps.

Let $I_{c}(x, y, t)$ be the camera image of the pattern generator (reflected on the surface) for time step $t$ (cf. Fig. 2B). Every camera pixel $(x, y)$ measures $n$ intensity values, originating from an unknown location on the screen; they can therefore be considered to be $n$ equidistant sample measurements of one period of a sinusoidal intensity function

$$
I_{c}(x, y, t)=A_{c}(x, y)+B_{c}(x, y) \cos \left(\Phi(x, y)-2 \pi \frac{t}{n}\right),
$$

where $A_{c}(x, y)$ is the mean intensity, $B_{c}(x, y)$ the intensity amplitude, and $\Phi(x, y)$ the phase of the sampled signal. ${ }^{14}$ Note that for a given $(x, y)$, Eq. (2) does not depend on information from any location $\left(x^{\prime}, y^{\prime}\right) \neq(x, y)$. 


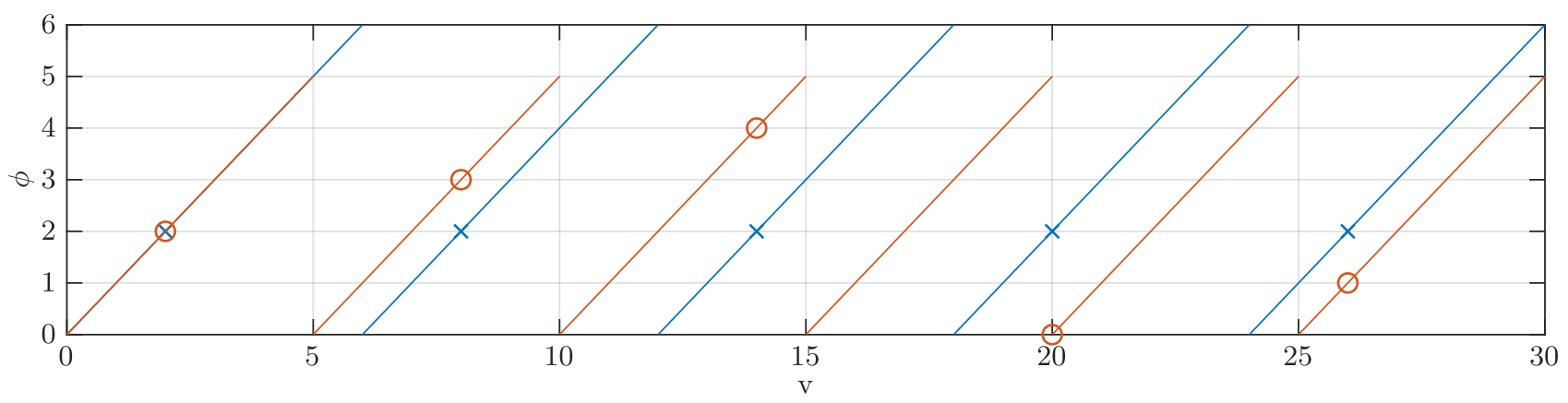

Figure 3. Two phase signals $\phi_{1}$ (blue) and $\phi_{2}$ (red) with coprime moduli of 6 and 5 pattern units, respectively, unambiguously encode the integer values in the interval [0,30). Each pair of integer values exists and occurs exactly once. For example, the red circles mark all occurrences of the phase value $\phi_{1}=2$ in the uniqueness interval, the blue crosses the corresponding values $\phi_{2}$; each blue cross marks a different value, and each integer in $[0,4]$ occurs once.

This local independence will remain true throughout the presented phase retrieval and phase unwrapping processes; the location variables $x$ and $y$ will therefore be omitted and every camera pixel considered independently in the following explanations. As there are three unknowns in Eq. (2), $n$ must be at least 3 ; it is often chosen to be 4 but can also be larger. The phase map $\Phi$ is computed as

$$
\Phi=\arctan \left(\frac{\sum_{t=0}^{n-1} I_{c}(t) \sin \left(2 \pi \frac{t}{n}\right)}{\sum_{t=0}^{n-1} I_{c}(t) \cos \left(2 \pi \frac{t}{n}\right)}\right) .
$$

The relation

$$
\phi=\omega \frac{\Phi}{2 \pi} \equiv v \quad(\bmod \omega)
$$

transforms the measured phase values $\Phi$ from radians into values $\phi$ relating to the pattern unit used in Eq. (1). Each such value is then equivalent (modulo $\omega$ ) to the coordinate $v$ of the screen location that is being imaged by the current camera pixel $(x, y)$. The process is repeated with a perpendicular fringe pattern to obtain the horizontal screen coordinates.

It is now obvious from Eq. (4), that $\phi$ immediately and unambiguously yields the required screen encoding, if the fringe width is chosen to be the same size as (or larger than) the screen dimension. Because the fringe widths used in practical applications are commonly one to two orders of magnitude smaller than the screen dimension, the retrieved phase maps are wrapped (cf.Fig. 2C) and, consequentially, the encoded screen locations ambiguous.

\subsection{Phase Unwrapping}

Consider first two (integer) coprime fringe pattern period widths $\omega_{1}$ and $\omega_{2}$. The Chinese remainder theorem ${ }^{15}$ states that for any pair of measured (integer) phase values $0 \leq \phi_{1}<\omega_{1}$ and $0 \leq \phi_{2}<\omega_{2}$, there exists one and only one $0 \leq v<\omega_{1} \omega_{2}$ that solves the linear congruences

$$
\begin{array}{ll}
\phi_{1} \equiv v & \left(\bmod \omega_{1}\right) \\
\phi_{2} \equiv v & \left(\bmod \omega_{2}\right)
\end{array}
$$

simultaneously. It is calculated as

$$
v=\left(\phi_{1}+\left(\phi_{2}-\phi_{1}\right) c_{1} \omega_{1} \bmod \omega_{1} \omega_{2}\right),
$$

where $c_{1}$ is an integer solution of the equation $c_{1} \omega_{1}+c_{2} \omega_{2}=\operatorname{gcd}\left(\omega_{1}, \omega_{2}\right)$ (gcd is the greatest common denominator, the coefficients $c_{1}$ and $c_{2}$ can be found with the well-known Euclidean algorithm ${ }^{15}$ ). Figure 3 plots the relation between $\phi_{1}, \phi_{2}$ and $v$ in the uniqueness range $\left[0, \omega_{1} \omega_{2}\right)$. Outside this interval, the measured phase value combinations become periodic. 
The biggest limitation of number-theoretical phase unwrapping with coprime period widths is its high susceptibility to phase noise (that comprises physical noise in the imaging system as well as sources of systematic errors $^{8}$ ). Small deviations of the measured phase values generally lead to large errors in the unwrapped phase values. ${ }^{14}$ Assume for instance a noise-induced error of 1 (screen pixel) in $\phi_{2}$. According to Eq. (7), this will result in an error of $\left(c_{1} \omega_{1} \bmod \omega_{1} \omega_{2}\right)$ screen pixels in the unwrapped phase value $v$ (for a noise-induced error of 1 screen pixel in $\phi_{1}$ the resulting error in $v$ is $\left(c_{1} \omega_{1}-1 \bmod \omega_{1} \omega_{2}\right)$ screen pixels).

It turns out that this limitation can be removed by using fringe patterns with non-coprime period widths. Consider two non-coprime period widths $\omega_{1}$ and $\omega_{2}$ that share a common cofactor $k$, i.e. $\omega_{1}=k \nu_{1}$ and $\omega_{2}=k \nu_{2}$ with two coprime base widths $\nu_{1}$ and $\nu_{2}$. What does this mean for phase unwrapping?

The first and obvious effect is that the range of unique unwrapped (integer) phase values that can be encoded is $k \nu_{1} \nu_{2}$ instead of $\omega_{1} \omega_{2}$ as in the case of coprime fringe periods. The order of the effectively usable pattern widths is therefore increased by a factor of $\sqrt{k}$, assuming a similar uniqueness range and ratio between the used period widths. Although the Chinese remainder theorem in its basic form requires coprime moduli, a generalization for two simultaneous linear congruences with non-coprime moduli exists. ${ }^{15}$ In effect Eq. (7) changes to

$$
v=\left(\phi_{1}+\frac{\left(\phi_{2}-\phi_{1}\right) c_{1} \omega_{1}}{k} \bmod k \nu_{1} \nu_{2}\right),
$$

but this only solves the system of Eqs. (5) and (6) under the precondition

$$
\phi_{1} \equiv \phi_{2} \quad(\bmod k)
$$

if Eq. (9) is violated, no solution exists. ${ }^{15}$

This immediately raises the question what this means for measurements $\left(\phi_{1}, \phi_{2}\right)$ that violate Eq (9) and how to proceed with them. If we ignore the mentioned precondition for the moment and just continue with Eq. (8), we find that there are now $k$ combinations of pairs of phase values $\left(\phi_{1}, \phi_{2}\right)$, instead of just one, that all correspond to the same unwrapped phase value $v$. However, only one of them corresponds to a measurement without phase noise and actually solves Eqs. (5) and (6), the others necessarily result from measurements affected by noise. This can be visualized in a $\omega_{1} \times \omega_{2}$ matrix structure, in which each entry corresponds to a specific measurement instance: the row is indexed by the first measured phase value $\phi_{1}$, the column by the second measured phase value $\phi_{2}$, and the matrix entry itself is the resulting unwrapped phase value $v$ (cf. Fig. 4).

All values $v$ that result from noise-free measurements lie on specific matrix diagonals. We term these matrix entries "legal", and all remaining matrix values "illegal"; as already noted, the latter do not actually solve Eqs. (5) and (6). This insight not only allows us to determine, whether or not a measurement triple $m=\left(\phi_{1}, \phi_{2}, v\right)$ is legal, but also to calculate a corrected, legal triple $m^{\prime}=\left(\phi_{1}^{\prime}, \phi_{2}^{\prime}, v^{\prime}\right)$, which is the legal matrix entry closest to $m$, measured perpendicular to the main diagonal direction (cf. Fig. 5). In order to calculate the corrected values $m^{\prime}$, we use Eq. (9) to discriminate the legal from the illegal measurements. Whenever Eq. (9) is violated, we distribute equal parts of the difference $\Delta \phi=\left(\phi_{2}-\phi_{1} \bmod k\right)$ to $\phi_{1}$ and $\phi_{2}$,

$$
\begin{aligned}
\phi_{1}^{\prime} & = \begin{cases}\phi_{1}+\frac{\Delta \phi}{2}, & \Delta \phi<\frac{k}{2} \\
\phi_{1}+\frac{\Delta \phi-k}{2}, & \Delta \phi \geq \frac{k}{2}\end{cases} \\
\phi_{2}^{\prime} & = \begin{cases}\phi_{2}-\frac{\Delta \phi}{2}, & \Delta \phi<\frac{k}{2} \\
\phi_{2}-\frac{\Delta \phi-k}{2}, & \Delta \phi \geq \frac{k}{2}\end{cases}
\end{aligned}
$$

$v^{\prime}$ is then the result of Eq. (8) when $\phi_{1}^{\prime}$ and $\phi_{2}^{\prime}$ are substituted for $\phi_{1}$ and $\phi_{2}$, respectively.

Fianlly, we will take a closer look at the quantitative properties of the results of the proposed phase correction technique. Consider therefore a triple $m^{*}=\left(\phi_{1}^{*}, \phi_{2}^{*}, v^{*}\right)$ of (unknown) noise-free wrapped and unwrapped phase values and $m=\left(\phi_{1}, \phi_{2}, v\right)=\left(\phi_{1}^{*}+n_{1}, \phi_{2}^{*}+n_{2}, v\right)$ a measurement of $m^{*}$ that is potentially affected by phase noise $n_{1}$ and $n_{2}$. Note that even legal measurements $m$ can be affected by noise, namely when $e_{1} \equiv e_{2}(\bmod k)$ (in this case, the equivalence in Eq. (9) remains unchanged by the noise). Figure 5 shows the banded structure that the proposed correction scheme imposes on the matrix representation of number-theoretical phase unwrapping. 


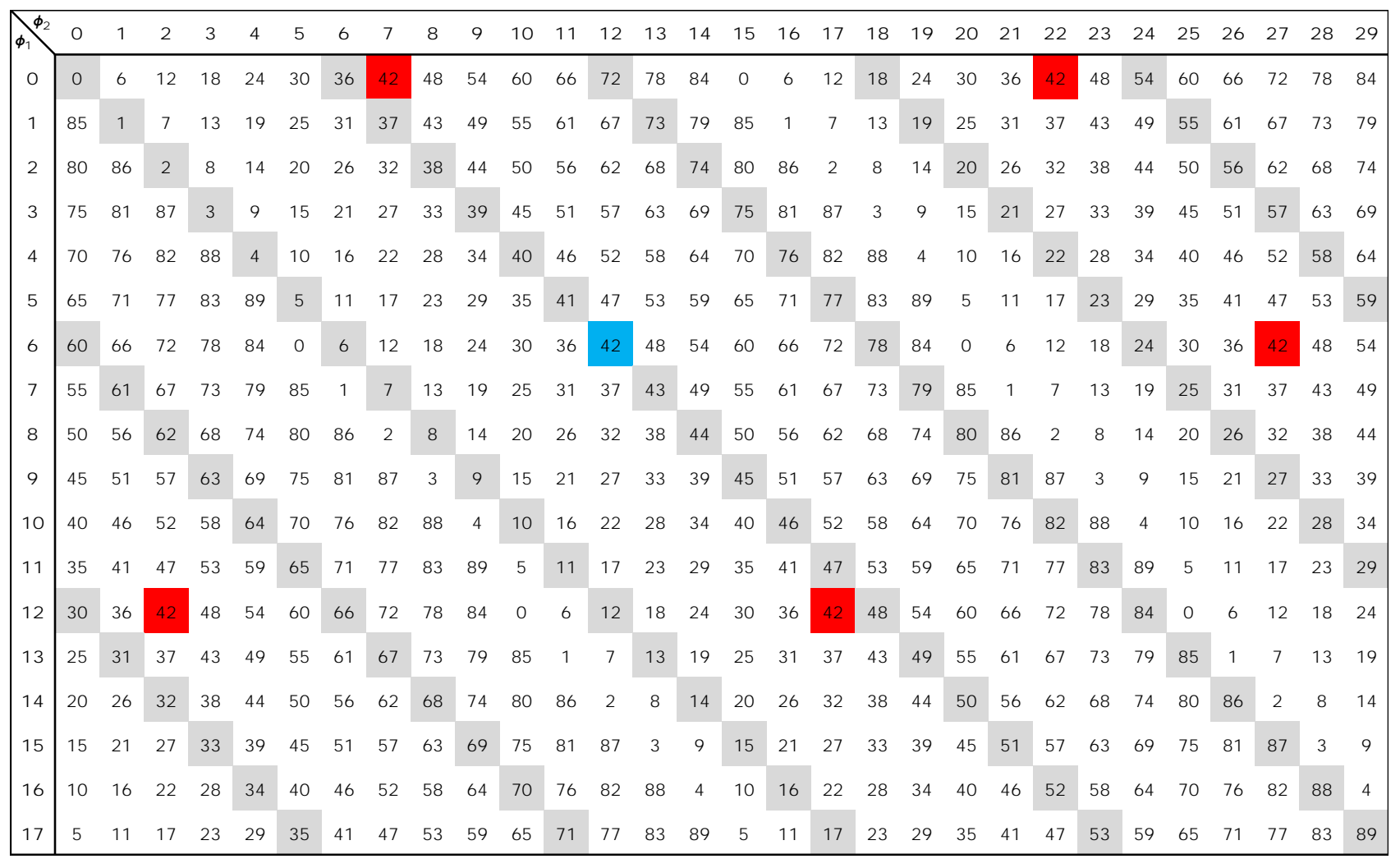

Figure 4. Matrix representation of two phase signals $\phi_{1}$ (row index) and $\phi_{2}$ (column index) with non-coprime moduli of 18 and 30 pattern units, respectively, and the unwrapped phase values $v$. The (greatest) common cofactor is $k=6$. Each unwrapped phase value occurs exactly $k$ times, but only one of these is "legal" (marked gray), all others are "illegal" (those terms are defined in the text). For instance, the blue matrix field marks the single legal entry for $v=42$, the red fields mark all illegal occurrences of that unwrapped phase value.

It is evident from Fig. 5, that the absolute unwrapped phase error $e_{v, a b s}=\left|v^{\prime}-v^{*}\right|$ remains limited as long as $m$ does not fall beyond a band border from $m^{*}$. More precisely, if the summed absolute phase errors remain below a threshold of $\left|e_{1}\right|+\left|e_{2}\right|<\frac{k}{2}$, the possible measurements $m$ form a square region around $m^{*}$ in the matrix representation. The resulting unwrapped phase error then also remains limited to $e_{v, a b s}<\frac{k}{2}$.

We have shown that the presented correction scheme is able to effectively limit the effect of noise-induced measurement errors up to a total noise level of $\frac{k}{2}$ with respect to the two original phase measurements. If the expected level of phase noise is known or can be estimated in advance, it is therefore possible to choose the factor $k$ accordingly.

It is worth noting that, despite being based inherently on integer arithmetics, number-theoretical phase unwrapping with sub-pixel precision for the phase values $\phi_{1}, \phi_{2}$ and $v$ is easily realized. To achieve a precision of $\frac{1}{f}$ of a screen pixel, $\phi_{1}$ and $\phi_{2}$ (as well as $\omega_{1}$ and $\omega_{2}$ ) are multiplied with the integer factor $f$ before transferring them to the the integer domain by rounding. Likewise the resulting unwrapped phase value is divided by $f$ to get $v$. This exemplifies the option to perform the phase retrieval and phase unwrapping computations in units other than screen pixels (see the beginning of Sec. 2.1).

\section{EXPERIMENTAL SETUP AND RESULTS}

In order to experimentally evaluate the proposed robust number-theoretical phase unwrapping method, we used reference phase maps created with the approach of exponentially decreasing fringe periods. This phase unwrapping technique, which has also been termed the multi-frequency approach, has previously been found to 


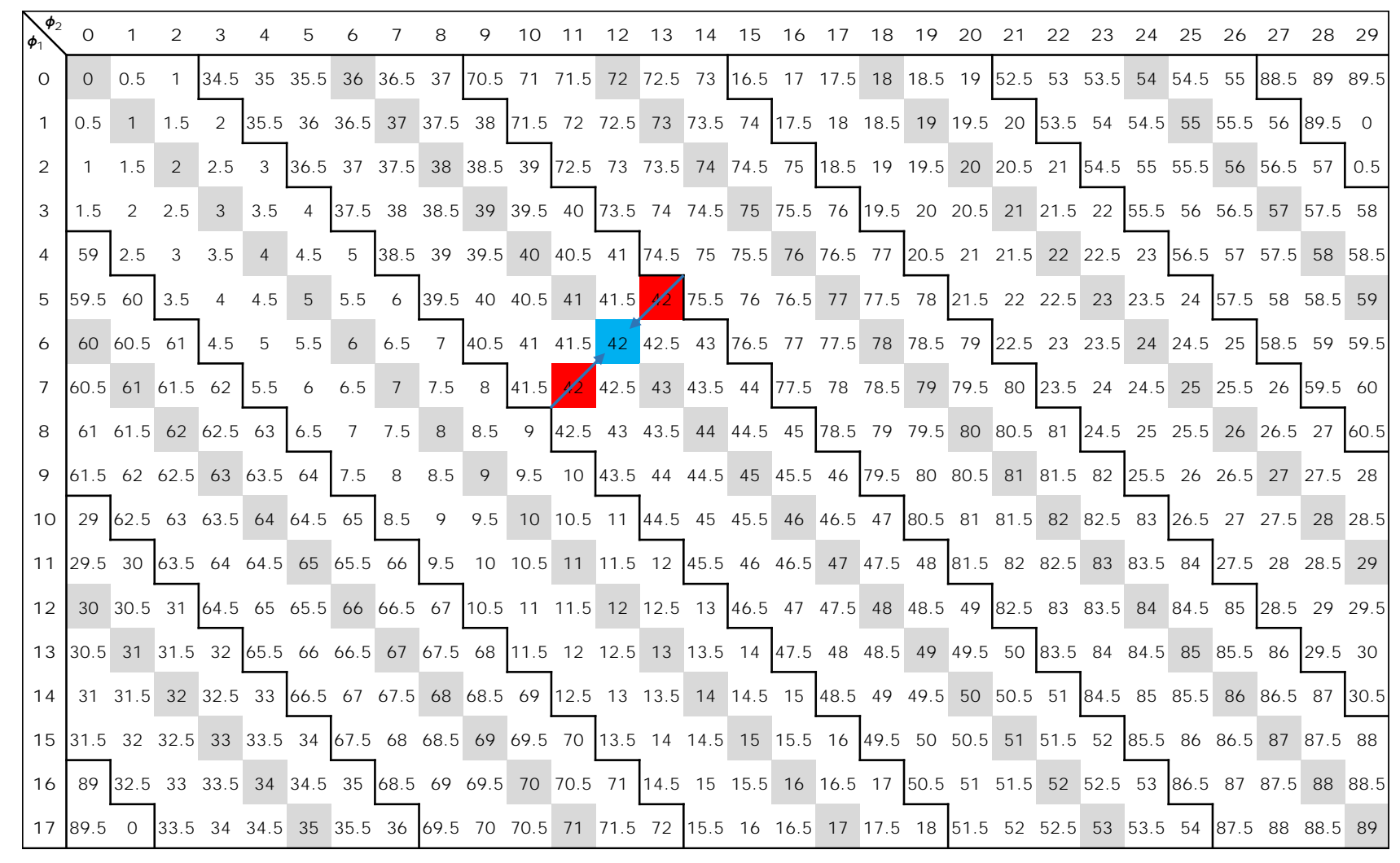

Figure 5. Correction of illegal measurements $\left(\phi_{1}, \phi_{2}, v\right)$ for the same parameters as used in Fig. 4; all unwrapped phase values $v$ not on the gray diagonals are substituted by the closest legal value, measured perpendicularly to the diagonals. For example the illegal matrix entries with red background have been substituted by the closest legal value $v=42$, marked blue.

outperform other approaches with regard to the quality of the resulting phase map. ${ }^{14}$ The screen we employed in the experimental setup has a pixel resolution of $3840 \times 2160$ pixels. We used fringe patterns with period widths of 4096, 1024 and 256 pixels for the multi-frequency approach; for the number-theoretical approach we chose period widths of 272 and 256 pixels. With $272=16 \cdot 17$ and $256=16 \cdot 16$, the common cofactor of the latter two period widths is $k=16$, the uniqueness range for phase unwrapping is therefore $\frac{272 \cdot 256}{16}=4352$ pixels. We deliberately chose identical smallest fringe pattern widths for both approaches, so that the local resolution of the resulting phase maps would be comparable. ${ }^{12}$ The test specimen was a planar front surface mirror. In addition to full screen pixels, the number-theoretical phase unwrapping was also carried out in units corresponding to pixel fractions of powers of 2 , ranging from $1 / 2$ of a screen pixel to $1 / 32$ of a screen pixel. No artificial limitation was imposed on the multi-frequency approach (i.e. the calculations were carried out using double precision floating-point numbers).

The experimental quantitative evaluation is based on the statistics of the differences $\Delta v=v_{n t}-v_{m f}$ between the unwrapped phase maps $v_{n t}$ of the number-theoretical approach and $v_{m f}$ of the reference multi-frequency approach. The histograms of the observed phase value deviations are presented in Fig. 6. Without the proposed correction technique, the mean absolute deviation of the resulting phase values was 1.99 pixels, with a high standard deviation of 5.43 pixels. As evidenced by Fig. 6 (top), this is attributable to deviation values around \pm 16 and \pm 17 pixels, which according to Eq. (8) are exactly the phase unwrapping errors that originate from phase noise of one pixel in the base signals $\phi_{1}$ or $\phi_{2}$ (there is also a single deviation value of approx. -32 pixels that results from a phase error of two pixels in one of the base signals).

An average of $10.9 \%$ of the measured data points were illegal according to our definition. Implementing the proposed correction scheme reduced the mean absolute deviation to 0.21 pixels with a standard deviation of 

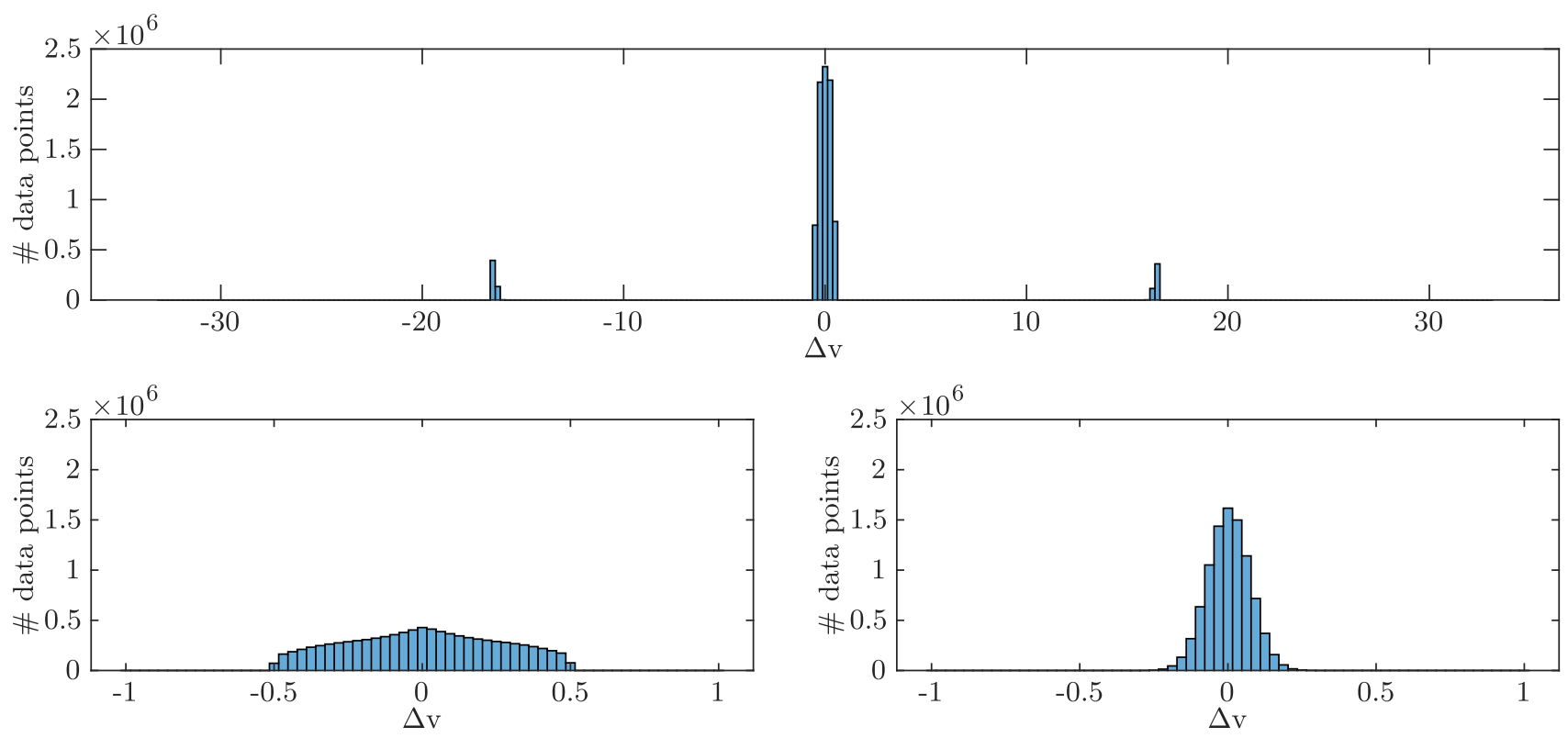

Figure 6. Histograms of the deviations of the number-theoretical phase unwrapping result values $v_{n t}$ from the reference phase values $v_{m f}$; (top) without correction, (bottom left) with the proposed correction with screen pixel resolution, (bottom right) with the proposed correction with sub-pixel resolution of 1/16 pixel.

0.26 pixels (cf. Fig. 6, bottom left); the highest observed absolute deviation from the reference was 0.91 pixels. Further improvement is achieved by going into the sub-pixel regime, decreasing the mean absolute deviation to 0.06 pixels and the standard deviation to 0.07 pixels at a precision of $1 / 16$ pixel (cf. Fig. 6, bottom right).

The effect of phase errors in $\phi_{1}$ or $\phi_{2}$ on the the unwrapping results can be seen in Fig. 7. It shows a 1D section from the center of the unwrapped phase map $v_{n t}$. Unwrapping errors that originate from illegal phase measurements manifest as spikes protruding upward or downward from the otherwise approximately linear signal. The spikes all possess the characteristic amplitude of around \pm 16 or \pm 17 pixels.

\section{CONCLUSIONS}

The presented technique extends and improves the number-theoretical phase unwrapping approach in PMD by adding the capability to detect and correct phase errors. Number-theoretical phase unwrapping is based on the mathematical concept of simultaneous linear congruences in modular arithmetics. The well-known Chinese remainder theorem tells us that such a system always has a solution (and this solution is unique in a certain interval) if all moduli are pairwise coprime. This changes if the premise of pairwise coprimality of the moduli is violated. The existence of a solution then becomes subject to the constraint given in Eq. (9). This constraint is exactly what makes discrimination between legal and illegal measurement tuples, and therefore phase error detection, possible when non-coprime fringe pattern widths are used. The capability to detect noise-induced phase errors comes at a cost, however, which is the reduced uniqueness range of the solution to the system of simultaneous linear congruences. If a required uniqueness interval (i.e. the screen dimension) is given, the pair of non-coprime fringe pattern widths will therefore necessarily be larger than if they could be chosen coprime.

The main contribution of the present paper is the introduction of the theoretical principles underlying the discrimination between legal and illegal pairs of base phase values from PMD measurements using fringe patterns with non-coprime fringe widths. Our experimental results show that the calculated phase maps are qualitatively comparable to those resulting with the multi-frequency approach. The presented phase unwrapping technique therefore combines the best of both worlds: The high robustness against phase noise and the ability for sub-pixel precision calculations from the multi-frequency approach and the lower number of required fringe pattern widths from the number-theoretic approach. Interestingly, the possibility of using non-coprime fringe widths for PMD 

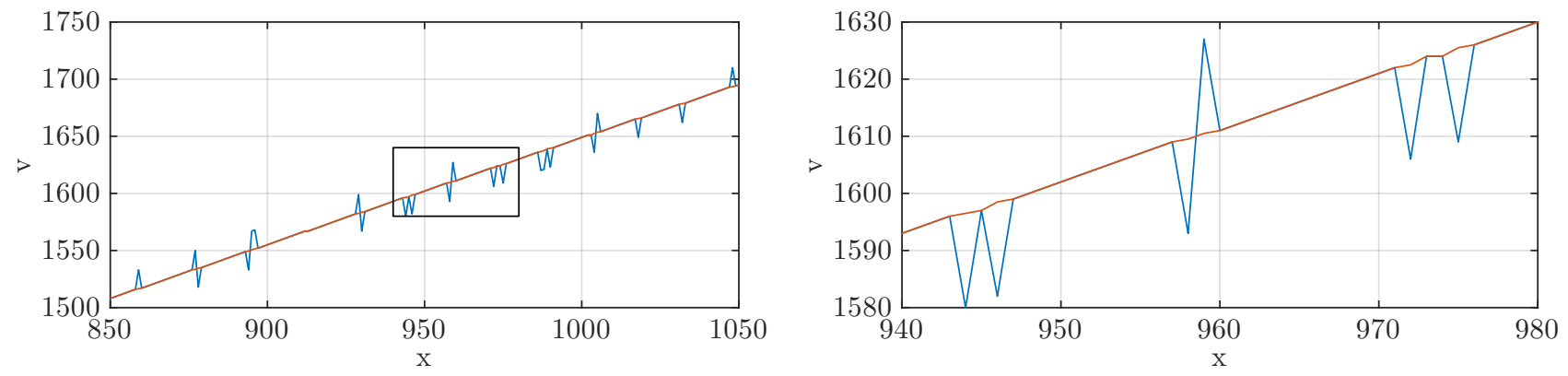

Figure 7. 1D section of the unwrapped phase map $v_{n t}$ with (red) and without (blue) the presented correction approach for illegal measurements. The right diagram shows a magnified plot of the black-boxed region in the left diagram.

has been mentioned in several previous publications, ${ }^{14,16}$ but to the best of our knowledge the potential for increased robustness against phase noise has not been exploited previously.

Future work should be dedicated into simulations of the proposed scheme in order to further support and quantify its error-correcting capabilities. Simulations will then also provide an excellent foundation to complement the experimental results presented in Sec. 3 with further comparative assessments of the improved number-theoretical approach and the multi-frequency approach against a perfectly known reference. Further improvements could be achieved by extending the presented method to more than two moduli, in order to decrease the possible fringe pattern widths. As a final note, although we develop the presented technique in the context of $\mathrm{PMD}$, it should be readily applicable to other phase-based metrological techniques such as 3D fringe projection profilometry.

\section{ACKNOWLEDGMENTS}

This work was funded by the programme Science and Technology of Nanosystems in the Research Field Key Technologies of the Helmholtz Association.

\section{REFERENCES}

[1] Gengenbach, U., Ungerer, M., Aytac, E., Koker, L., Reichert, K.-M., Stiller, P., and Hagenmeyer, V., "An integrated workflow to automatically fabricate flexible electronics by functional printing and smt component mounting," in [2018 IEEE 14th International Conference on Automation Science and Engineering (CASE 2018)], 1624-1629, IEEE (2018).

[2] Baby, T. T., Cadilha Marques, G., Neuper, F., Singaraju, S. A., Garlapati, S., von Seggern, F., Kruk, R., Dasgupta, S., Sykora, B., Breitung, B., Anitha Sukkurji, P., Bog, U., Kumar, R., Fuchs, H., Reinheimer, T., Mikolajekh, M., Binder, J. R., Hirtz, M., Ungerer, M., Koker, L., Gengenbach, U., Mishra, N., Gruber, P., Tahoori, M., Aghassi Hagmann, J., von Seggern, H., and Hahn, H., "Printing technologies for integration of electronic devices and sensors," in [Functional Nanostructures and Sensors for CBRN Defence and Environmental Safety and Security (FNS-CBRN Defence - 2018)], NATO Science for Peace and Security Series C: Environmental Security, Springer, Dordrecht (in print).

[3] Knauer, M. C., Kaminski, J., and Häusler, G., "Phase measuring deflectometry: a new approach to measure specular free-form surfaces," in [Optical Metrology in Production Engineering], Osten, W. and Takeda, M., eds., Proc. SPIE 5457, 366-376, SPIE (2004).

[4] Huang, L., Idir, M., Zuo, C., and Asundi, A., "Review of phase measuring deflectometry," Opt. Lasers Eng. 107, 247-257 (2018).

[5] Park, H., Hong, D., and Cho, H., "A two dimensional phase-shifting method for deflectometry," in [Optomechatronic Technologies 2008], Otani, Y., Bellouard, Y., Wen, J. T., Hodko, D., Katagiri, Y., Kassegne, S. K., Kofman, J., Kaneko, S., Perez, C. A., Coquin, D., Kaynak, O., Cho, Y., Fukuda, T., Yi, J., and Janabi-Sharifi, F., eds., Proc. SPIE 7266, 72661L, SPIE (2008). 
[6] Flores, J. L., Bravo-Medina, B., and Ferrari, J. A., "One-frame two-dimensional deflectometry for phase retrieval by addition of orthogonal fringe patterns," Appl. Opt. 52, 6537-6542 (2013).

[7] Liu, Y., Olesch, E., Yang, Z., and Häusler, G., "Fast and accurate deflectometry with crossed fringes," Adv. Opt. Technol. 3, 441-445 (2014).

[8] Faber, C., [New Methods and Advances in Deflectometry], Erlangen Scientific Press, Erlangen (2012).

[9] Kaminski, J., [Geometrische Rekonstruktion spiegelnder Oberflächen aus deflektometrischen Messdaten], Lehrstuhl für Mikrocharakterisierung, Erlangen (2008).

[10] Beyerer, J., Puente León, F., and Frese, C., [Machine Vision - Automated Visual Inspection: Theory, Practice and Applications], Springer, Berlin (2016).

[11] Petz, M. and Ritter, R., "Reflection grating method for 3d measurement of reflecting surfaces," in [Optical Measurement Systems for Industrial Inspection II: Applications in Production Engineering], Hö̈ling, R., Jüptner, W. P. O., and Kujawinska, M., eds., Proc. SPIE 4399, 35-41, SPIE (2001).

[12] Fischer, M., [Deflektometrie in Transmission - Ein neues Messverfahren zur Erfassung der Geometrie asphärischer refraktiver Optiken], Shaker, Aachen (2016).

[13] Ghiglia, D. C. and Pritt, M. D., [Two-Dimensional Phase Unwrapping - Theory, Algorithms, and Software], Wiley, New York, NY (1998).

[14] Zuo, C., Huang, L., Zhang, M., Chen, Q., and Asundi, A., "Temporal phase unwrapping algorithms for fringe projection profilometry: A comparative review," Opt. Lasers Eng. 85, 84-103 (2016).

[15] Takloo-Bighash, R., [A Pythagorean Introduction to Number Theory - Right Triangles, Sums of Squares, and Arithmetic], Springer, Cham (2018).

[16] Knauer, M. C., [Absolute Phasenmessende Deflektometrie], Lehrstuhl für Mikrocharakterisierung, Erlangen (2006). 